\title{
Jojoba oil: Anew media for frying process
}

\author{
Shaker M Arafat ${ }^{1}$ and Amany M Basuny*2 \\ ${ }^{1}$ Department of Oils \& Fats Research, Food Technology Research Institute, Egypt \\ ${ }^{2}$ Department of Biochemisrty, Agriculture Beni-Suef University, Egypt
}

Submission: September 17, 2018; Published: October 12, 2018

*Corresponding author: Amany M Basuny, Department of Oils \& Fats Research, Food Technology Research Institute, Agricultural Research Center, Egypt, Email: dr_shakerarafat@yahoo.com,dramany_basuny@yahoo.com

\section{Introduction}

Jojoba Oil health benefits includes reducing signs of premature aging, an excellent moisturizer, helps fade stretch marks, helps fight fungal, helps minimize breakouts, prevent razor burns and bumps, help speed up wound healing, can help prevent chapped lips, stimulate hair growth, assisting managing psoriasis and eczema, aid in healing sunburns, and helps treat dry scalp. If you've ever used any kind of cosmetic product, chances are it contained some sort of Jojoba oil extract. Jojoba oil is extracted from the seed of the Simmondsia chinensis (or simply Jojoba plant), a shrub that grows in regions of California, Arizona and northern Mexico. Jojoba oil is not a recent discovery, as there is evidence that the Native Indians living in these regions used Jojoba oil centuries ago to treat wounds and numerous other ailments. It is also interesting to note that Jojoba "oil", is not really an oil, but rather a liquid wax. This separates it from other common oils in the sense that they have high triglyceride content, which contributes to their instability (or explains why they go rancid very fast). Notable exceptions are coconut and castor oils, which have high triglyceride content but are extremely stable as well.

Jojoba (pronounced ho-ho-ba) oil is a vegetable oil obtained from the crushed bean of the jojoba shrub (Simmondsia chinenis). The jojoba shrub is native to the Sonoran Desert of northwestern Mexico and neighboring regions in Arizona and southern California. It grows in dense stands throughout that region. The woody evergreen shrub may reach $15 \mathrm{ft}$ ( $4.5 \mathrm{~m}$ ) in height. Jojoba has flat gray-green leathery leaves and a deep root system that make it well adapted to desert heat and drought. It has a life span of 100-200 years, depending on environmental conditions. Jojoba grows best in areas with $10-18$ in $(25-45 \mathrm{~cm})$ of annual rainfall where temperatures seldom fall below $25^{\circ} \mathrm{F}\left(-4^{\circ} \mathrm{C}\right)$ for more than a few hours at night. It can grow on many types of soils, including porous rocks, in slightly acid to alkaline soils, and on mountain slopes or in valleys [1].

Jojoba shrubs are dioecious, meaning plants are either male (staminate), producing pollen, or female (pistillate), producing flowers. The small flowers have no odor or petals and do not attract pollinating insects. The flowers are pollinated by wind in late March; the flowers develop into fruit by August, with full maturation occurring by October. The green fruit dries in the desert heat, its outer skin shriveling and pulling back to expose a wrinkled brown soft-skinned seed (referred to as a nut or bean) the size of a small olive [2]. These nuts, which resemble coffee beans, contain a vegetable oil that is clear and odorless but less oily to the touch than traditional edible oils. The oil comprises half of the weight of the nut. There are about 1,700 seeds in a pound; $17 \mathrm{lb}(6.3 \mathrm{~kg})$ of jojoba seeds are required to produce one gallon of oil.

Native Americans have used jojoba for hundreds of years. In the 1700s, Father Junipero Serra, the founder of 21 California missions, noted in his diary that the Native Americans were using the oil and the seeds for many different purposes: for treating sores, cuts, bruises, and burns ; as a diet supplement and as an appetite suppressant when food was not available; as a skin conditioner, for soothing windburn and sunburn ; as a cooking oil; as a hair or scalp treatment and hair restorative; and as a coffeelike beverage by roasting the seeds.

The chemical structure of jojoba oil is different from that of other vegetable oils. Rather than being an oil, it is actually is a polyunsaturated liquid wax that is similar to sperm whale oil, though without the fishy odor. It is made of fatty acids as well as esters composed entirely of straight chain alcohols. Both the acid and alcohol portions of jojoba oil have 20 or 22 carbon atoms, and each has one unsaturated bond. Waxes of this type are difficult to synthesize. As a wax, jojoba oil is especially useful for applications that require moisture control, protection, and emolliency. Jojoba oil is liquid at room temperature because of its unsaturated fatty acids. It does not oxidize or become rancid and does not break down under high temperatures and pressures. Jojoba oil can be heated to $370^{\circ} \mathrm{F}\left(188^{\circ} \mathrm{C}\right)$ for 96 hours without exhibiting degradation in general composition and carbon chain length. The stability shown by jojoba oil makes it especially useful for cosmetic applications.

When the United States banned the use of sperm whale oil (spermaceti wax) in 1974, the government began to fund efforts to investigate and cultivate jojoba as a replacement. Jojoba oil was found to be an adequate substitute for applications that had previously used sperm whale oil. Jojoba oil has many uses in a wide variety of industries. As a cosmetic, it is an effective cleanser, 
conditioner, moisturizer, and softener for the skin and hair. It is applied directly to the skin to soften the skin, to reduce wrinkles and stretch marks, to lighten and help heal scars, and to promote healthy scalp and hair. Jojoba oil is similar to, and miscible with, sebum, which is secreted by human sebaceous glands to lubricate and protect skin and hair. When sebum production decreases due to age, pollutants, or environmental stresses, jojoba oil can be used to replicate sebum oil. Jojoba oil can accumulate around hair roots, thereby conditioning hair and preventing it from becoming brittle and dull. If there is too much sebum buildup on the scalp, it dissolves and removes the sebum, leaving the hair clean. Jojoba oil as a solubilizing agent can also remove sticky buildup on hair from hair preparations as well as airborne particulates deposited on the hair. It forms a lipid layer on the skin, acting as a moisturizer, as well as penetrating and being absorbed by the outer layer of skin. It is widely used as an ingredient in shampoos, conditioners, facial, hand and body lotions, cuticle and nail care products, baby care lotions, creams and oils, cleansers, moisturizers, bath oils and soaps, sunscreen lotions, and makeup products. Jojoba oil is also used as a base in the manufacture of perfume. The potential therapeutic uses of jojoba oil include the treatment of acne, cold sores, and such skin diseases as psoriasis.

Jojoba oil is also a registered (licensed for sale) pesticide for use on crops. It is used to control white flies on all crops and powdery mildew on grapes and ornamentals. It is applied as a spray containing $1 \%$ or less final concentration of jojoba oil. It acts as a pesticide by forming a physical barrier between an insect pest and the leaf surface. Because of its low toxicity and its rapid degradation in the environment, jojoba oil does not pose a risk to non-target organisms or the environment; though as an oil, it should not be disposed of in lakes or other bodies of water.

Jojoba oil is prepared by pressing the jojoba seeds to extract the oil, followed by filtration. It is then pasteurized to ensure product safety. Four grades of jojoba oil are produced:

i. A pure, natural golden grade, a golden-yellow color oil that is produced by the basic production process;

ii. Refined and bleached jojoba oil, with color removed by bleaching and filtration;

iii. A decolorized/deodorized grade, which is used in cosmetics requiring colorless and odorless oils;

iv. A molecular distilled grade, an expensive formulation produced in minimal quantities, with its use having mostly been replaced with decolorized/deodorized jojoba oil.

Jojoba oil is a nontoxic, noncomedogenic (does not clog pores), and hypoallergenic substance. It has been widely used for decades in cosmetics, with no reported adverse effects. If jojoba oil is ingested, most of it is eliminated in the feces, with little getting distributed in the body. Jojoba oil seeds of the shrub Simmondsia chinensis ( $S$. californica) have long been used as food by native Americans and roasted and ground to prepare beverages. The oil is a liquid wax of long-chain fatty acids (eicosenoic and decosenoic (erucic) acids) esterified with long-chain alcohols (eicosanol and docosanol). Of interest in cosmetics as a replacement for sperm whale oil but also has food applications, e.g. as a coating agent for dried fruits [3]. Deep-fat frying of foods is considered everywhere in the world to be the most common unit operation used in food preparation. Deep-fat frying indicates he ating oil or fat in the presence of moisture and air and then undergoes extensive degradation through oxidation. This process comprises oxidation of the unsaturated centres of the unsaturated fatty acids and the resultant hyperoxides, carcinogenic substances, will decompose to produce in general volatile substances such as aldehydes, ketones and acids, the compounds responsible for the off-flavour. In this sit uation, several methods are used to prevent or delay oil oxidation and improve the quality of fried oils. These methods include: methyl siloxane, blending or mixing oils (mixing higholeic sunflower oil with other oils), membrane process, ferrous sulphate [4].

The people of Egypt are fond of fried foods such as falafal, potato chips, French fries, fish sticks and fried chicken. Therefore, large amounts of oils are needed to cope up with the high consumption of oils for deep-fat frying. Because of the shortage of oil production, alternative ways are need ed to overcome this situation; among these ways is to find another heating medium for deep-fat frying. In general, the deep-fat frying medium has to fulfill the following characteristics: non-glyceride substance (low caloric compounds), does not smoke above $200^{\circ} \mathrm{C}$, withstand thermal oxidation and cause no nutritional risks.

The jojoba oil is an edible oil rich in all sorts of vitamins and minerals which is what causes its dense physical appearance. It is literally saturated in them, which is why so many industries today can find so many uses for it. There are even refined and organic jojoba oil currently for sale, similar to the cooking grade categories of other famous cooking oils such as the olive oil. The jojoba oil can also withstand high temperatures without dissociating. So, can the jojoba oil be used for cooking? The short answer is yes, but it isn't recommended to do so at all. The different categories and grade of the jojoba oil sold today are only meant for external and topical applications and not for consumption [5]. Its use in the culinary industry remains limited because it is expensive compared to other mainstream cooking oils due to its limited production rate [5].

When consumed, the jojoba oil will not provide any calories because it cannot be digested by our body but instead act as a lubricant for our digestive system. This means that all of its nutritious content will remain in the oil, making it practically useless for us. People looking to control their calorie intake are using it as a diet oil since it does not provide any calories. However, taking in too much of the jojoba oil will cause steatorrhea, a condition where excess fats appear in the stools. If at all you decide to use the jojoba oil as a cooking oil, make sure you use the organic and pure version of it to avoid consuming impurities or other artificial compounds. Also, take small and controlled amounts of the jojoba oil to avoid steatorrhea. Although its use today in the culinary industry remains limited, jojoba oil continues to thrive in other industries due to its versatility and medicinal properties. 
The oil is composed mainly of straight chain monoesters in the range of $\mathrm{Cl} 8-\mathrm{C} 24$ as alcohol s and acids with two double bonds, one at each side of the ester bond $[6,7]$. The human lipase enzymes, which digest most vegetable oils and animal fats, do not digest jojoba oil, allowing it to pass through the digestive tract largely unmetabolised. In addition, Anantharaman [8] reported that jojoba oil is slow to turn rancid and undamaged by heating to high temperatures. Furthermore, Isbell et al. (1999) studied the oxidative stability indices of several vegetable oils at $110^{\circ} \mathrm{C}$. They reported that crude jojoba oil possessed good oxidative stability. Beneficial results were also demonstrated in studies focusing on replacing edible fats and oils by jojoba oil in margarine and mayonnaise.

Concerning the folk medicine uses of jojoba oil, it was reported to have a wide range of uses. Indians of California used the oil as a medicine for cancer, kidney disorders, headache, wounds and sore throat. Also, the oil was reported to be used in cosmetics as antidandruff, in hair-restore, hair creams and body lotions. Furthermore, jojoba oil was found to be effective in treatment of acne, eczema and psoriasis. Also, the oil possesses an anti-microbial, anti- parasitic, anti-pyretic, antiinflammatory [9] and hypocholesterolemic effects. Several applications have been reported, including coating of foodstuffs, which greatly improved stability in comparison with hydrogenated oils.

Mineral hydrocarbons (MHC) are derived from petroleum sources. Structurally, the hydrocarbons found in mineral oils are mostly paraffins (alkanes of straight chains), isoparaffins (branched chains) and ycloparaffins (saturated rings with side chains). The Scientific Committee on Food has assessed the toxicity of mineral and synthetic Furthermore, jojoba oil was found to be effective in treatment of acne, eczema and psoriasis. Also, the oil possesses an anti-microbial, anti- parasitic, antipyretic, anti-inflammatory [9] and hypocholesterolemic effects. Several applications have been reported, including coating of foodstuffs, which greatly improved stability in comparison with hydrogenated oils. Mineral hydrocarbons (MHC) are derived from petroleum sources. Structurally, the hydrocarbons found in mineral oils are mostly paraffins (alkanes of straight chains), isoparaffins (branched chains) and cycloparaffins (saturated rings with side chains). Application of MHC and petroleum are intentionally applied to both direct and indirect food additive uses. Some approved direct additive uses of MHC as coatings for fruits and vegetables, sealing and polishing agents for confectionary, dust-control agents for grains during storage and as grease and pan-release oils in baking [10]. Indirect additive applications of MHC include uses as components of carton and packaging materials, adhesives, food-grade lubricants and sealants for bottles and home-canning jarscant. In addition, MHC are widely used in cosmetics and pharmaceutical products [11].

\section{References}

1. Baldwin AR (1989) Seventh International Conference on Jojoba and Its Uses. American Oil Chemists Society pp. 453.

2. Wisniak J (1987) The Chemistry and Technology of Jojoba Oil. American Oil Chemists Society.

3. Eltaweel AA, Aly AA, El-Bolok TK, Shaker M Arafat (2017) Evaluation of some female jojoba genotypes under sandy land condition. J Plant Production Mansura Univ 8(8): 877-885.

4. Farag RS, Mostafa MF, Rehab FMA (2008 a) Use of sunflower oil mixed with jojoba and paraffin oils in deep-fat frying process. International Journal of Food Science and Technology 43(7): 1306-1315.

5. Farag, R. S., Mostafa, M. F., Amany, M. M. B. and Rehab F. M. (2008 b) Safety evaluation of individual non fried and fried sunflower oil, paraffin oil, jojoba oil and their binary mixture on rat health. International Journal of Food Science and Nutrition 43(10): 1742-1753.

6. Saguy IS, Shani A, Weinberg P, Garti N (1996) Utilization of jojoba oil for deep-fat frying of foods. Lebensmittel-Wissenschaft und-Technologie 29(5-6): 573-577.

7. Abbott TP, Holser RA, Plattner BJ, Plattner RD, Purcell HC (1999) Pilot-scale isolation of simmondsin and related jojoba constituents. Industrial Crops and Products 10(1): 65-72.

8. Anantharaman K (1994) Jojoba oil - nutritional and safety studies and potentials in clinical nutrition. IX International Conference on Jojoba and its Uses, Catamarca, Argentina.

9. Hanan MS, Elham AM (1996) Some biological and pharmaceutical studies on jojoba oil. Bulletin of Faculty of Pharmacy, Cairo University 34: 239-243.

10. MHW (1992) The Japanese Standard of Food Additives. (6 $6^{\text {th }}$ edn), Tokyo pp. 558.

11. Tennant DR (2004) The usage, occurrence and dietary intakes of white mineral oils and waxes in Europe. Food Chemistry and Toxicology 42(3): 481-492.

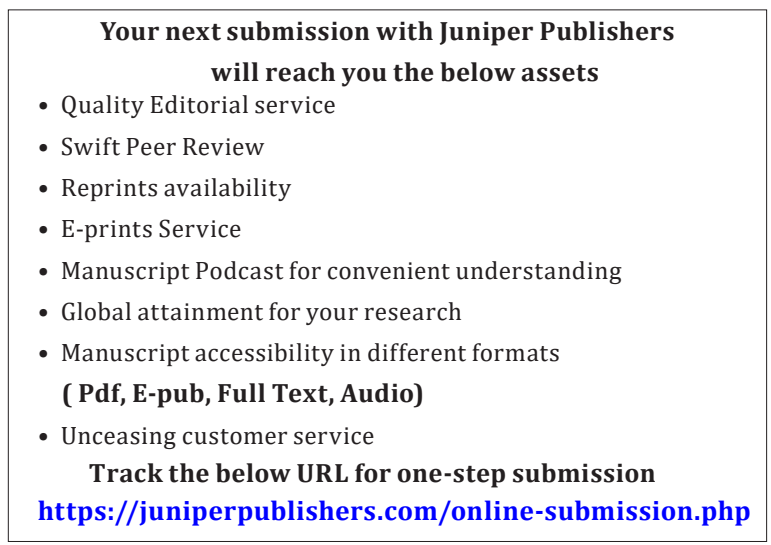

